Cooper, A.K., O’Brien, P.E., and Richter, C. (Eds.)

Proceedings of the Ocean Drilling Program, Scientific Results Volume 188

\title{
Data Report: Multisensor CORE LogGing Data, CoArse-Fraction Grain-Size AnAlyses, and BIOGENIC Silica CONTENT OF UPPER MIOCENE- LOWER Pliocene Sediments, ODP SITE 1165'
}

Jens Grützner²

\section{INTRODUCTION}

The Prydz Bay area is a key region for studying and understanding the history of the eastern Antarctic Continental Ice Sheet ( $\mathrm{O}^{\prime}$ Brien, Cooper, Richter, et al., 2001). Ocean Drilling Program (ODP) Site 1165 is situated in a water depth of $3357 \mathrm{~m}$ on the continental rise offshore from Prydz Bay and lies in front of the outlet for the Lambert GlacierAmery Ice Shelf system that today drains $22 \%$ of East Antarctica. The site was drilled into mixed pelagic and hemipelagic sediments from the southwestern side of the Wild Drift. The drift is an elongate sediment body formed by the interaction of sediment supplied from continental shelf and slope with westward-flowing bottom currents. The sedimentary sequence is characterized by alternations between a generally gray to dark gray facies and a green to greenish gray facies. The greenish facies are structureless diatom-bearing clays with common bioturbation and larger amounts (>15\%-20\%) of biogenic silica, dispersed clasts, and lonestones than the dark gray facies, which are mostly less bioturbated clay with some silt laminations (Shipboard Scientific Party, 2001). Highquality advanced piston corer and extended core barrel cores containing nearly complete sections of middle Miocene to early Pliocene age allow a detailed characterization of sedimentary cycles and can provide
${ }^{1}$ Grützner, J., 2003. Data report: Multisensor core logging data, coarsefraction grain-size analyses, and biogenic silica content of upper Miocene-lower Pliocene sediments, ODP Site 1165. In Cooper, A.K., O'Brien, P.E., and Richter, C. (Eds.), Proc. ODP, Sci. Results, 188, 1-16 [Online]. Available from World Wide Web: <http://www-odp.tamu.edu/ publications/188_SR/VOLUME/ CHAPTERS/007.PDF>. [Cited YYYYMM-DD]

${ }^{2}$ Bremen University, Geosciences Department, PO Box 3304 40, 28334 Bremen, Germany.

jgruetzn@allgeo.uni-bremen.de

Initial receipt: 17 July 2002

Acceptance: 12 December 2002

Web publication: 11 March 2003

Ms 188SR-007 


\section{J. GRÜTZNER}

Data Report: Core Data, Grain-Size Analysis, and Biogenic Silica

indications for ice advances of the Lambert Glacier system into Prydz Bay, for the extent of sea ice, and for changes in oceanic circulation.

The purpose of this work is to provide a data set of coarse-fraction mass percentage ( $>63,>125$, and $>250 \mu \mathrm{m}$ ) and biogenic silica content measured on sediments of late Miocene to early Pliocene age drilled at Site 1165. Additionally, high-resolution records of magnetic susceptibility (MS) and gamma ray attenuation (GRA) bulk density are presented. These shipboard data sets were edited postcruise. Furthermore, I provide a high-resolution dry bulk density record that is derived from GRA bulk density and can be used for the calculation of mass accumulation rates. These sedimentological and physical parameters will be used in future work to understand the depositional pattern of alternating biogenic and terrigenous sediments that was observed at Site 1165 (Shipboard Scientific Party, 2001).

\section{METHODS}

The weight percentage of the bulk parameter $>63 \mu \mathrm{m}$ (coarse fraction) was measured on 274 samples from Cores 188-1165B-4H through $14 \mathrm{H}$ and Core $188-1165 \mathrm{C}-7 \mathrm{R}$. The sample resolution is $30 \mathrm{~cm}$, equivalent to a time resolution of 5 to 15 k.y., depending on sedimentation rate. All bulk samples were freeze dried, and the total mass of the bulk sample was determined. No further disaggregation procedure was used. Sand content was determined after wet sieving at $63 \mu \mathrm{m}$. The $>63-\mu \mathrm{m}$ samples were wet sieved into three subsamples (63-125, 125-250, and 250-500 $\mu \mathrm{m}$ ). The sand subsamples were dried and weighed.

Splits of 76 samples (1-2 per section) used for grain-size analyses were also measured for opal-A content. Homogenized dry bulk samples were analyzed using an automated leaching technique after Müller and Schneider (1993), which was shown to be reliable in an interlaboratory comparison (Conley, 1998). Silica was extracted by wet chemical means with 1-M NaOH, and concentrations of dissolved silica were measured by molybdate blue spectrophotometry. The proportion of biogenic silica was determined by graphical analysis of the absorbance vs. time plot (DeMaster, 1981). Resulting silica values were converted into weight percent biogenic $\mathrm{SiO}_{2}$ and are given as weight percent biogenic silica by assuming a uniform bound water content of $10 \mathrm{wt} \%$ within the opaline substance. According to Müller and Schneider (1993), the relative accuracy of the method is better than $2 \%$ for samples rich in biogenic silica and $4 \%-10 \%$ for samples with $<10 \mathrm{wt} \%$ biogenic silica, respectively. Bonn et al. (1998) assumed an artificial background value of 1-2 wt\% "pseudo-opal," caused by a partial leaching of clay minerals. In contrast, Hillenbrand and Fütterer (2001) measured opal contents down to $0.4 \mathrm{wt} \%$ on discrete samples, suggesting a negligible background opal signal. No correction was applied to the opal data reported here.

Data sets of magnetic susceptibility and bulk density measured with the shipboard multisensor track (MST) on Cores 188-1165B-1H through $17 \mathrm{H}$ and Core 188-1165C-7R (Shipboard Scientific Party, 2001) have been edited for erroneous measurements resulting from section breaks and voids. Section 188-1165-11H-5, which was not run on the MST during the cruise, was measured using a split-core logger located in the ODP core repository. Because of different measurement conditions, shore-based (MS point sensor, split cores, and dryer sediment) and shipboard (MS loop sensor, whole cores, and fresh sediment) corrections had to be made to the shore-based data sets. This was accomplished by 


\section{J. GRÜTZNER}

remeasuring Section 188-1165-11H-6 with the split-core logger and from this deriving a linear correlation between ship- and shore-based data sets. The GRA bulk densities $\left(\rho_{\mathrm{GRA}}\right)$ were converted into dry bulk densities $\left(\rho_{\text {dry }}\right)$ using the equation

$$
\rho_{\text {dry }}=-1.5228+1.5432 \times \rho_{\mathrm{GRA}} .
$$

The linear relationship between wet bulk density and dry bulk density measurements is derived from shipboard moisture and density measurements performed on discrete samples (Fig. F1). To quantify the correlation between the different parameters, the MST data were resampled at the exact depths for which grain size and opal-A data are available.

\section{RESULTS}

Bulk sediment parameters (grain size and biogenic silica), edited MST measurements, and calculated dry density are presented in Tables T1, T2, T3, and T4 and plotted vs. depth in Figures F2 and F3. Measurement statistics and linear correlation coefficients between the parameters are given in Tables T5 and T6. The general downhole trend of MST records was already described in the Leg 188 Initial Reports volume (O'Brien, Cooper, Richter, et al., 2001). Thus, I here focus only on the sedimentological parameters and the comparison of these new records with the MST data sets.

The average sand content ( $>63 \mu \mathrm{m})$ over the investigated interval is relatively low (8\%), indicating a low-energy depositional environment. However, prominent spikes of up to $30 \%$ sand occur. A separation of the size classes (Fig. F2) reveals that these events are largely controlled by ice-rafted detritus (IRD) of $>250 \mu \mathrm{m}$ size. The $>250-\mu \mathrm{m}$ record shows only a moderate positive correlation $(r=0.47)$ with magnetic susceptibility and a weak negative $(r=-0.14)$ correlation with density (Table T6). However, every IRD peak (more than $10 \%>250 \mu \mathrm{m}$ ) is reflected by sharp positive spikes in the magnetic susceptibility and density records (Fig. F3). It is likely that other sharp spikes observed in the MS data also represent IRD layers that were missed by the $30-\mathrm{cm}$ spacing of the grain-size samples.

Biogenic silica content varies between $4.5 \%$ and $32.7 \%$, with an average of $17.1 \%$. A moderately negative correlation $(r=-0.51)$ of biogenic silica and bulk density exists. Higher biosiliceous content above 50 mbsf is reflected by a zone of lower density $\left(\sim 1.50 \mathrm{~g} / \mathrm{cm}^{3}\right)$ that stretches from 35 to 50 mbsf. Furthermore, biogenic silica is positively correlated to the $>250-\mu \mathrm{m}$ record $(r=0.27)$, which confirms the observation that IRD input is more abundant in greenish (diatom rich) intervals (Shipboard Scientific Party, 2001)

A comparison of IRD $(>250 \mu \mathrm{m})$ and biogenic silica content vs. age is shown in Figure F4. The depth to age conversion (Table T7) is based on shipboard bio- and magnetostratigraphy (Shipboard Scientific Party, 2001). During the late Miocene (9-5 Ma) IRD fluctuations are superimposed on a general trend to higher IRD input, which is followed by a trend to lower IRD content during the early Pliocene (5-3.5 Ma) and a marked decrease at 3.5 Ma. The biogenic silica time series exhibits variations around a $15 \%$ average during the late Miocene followed by a significant low (5\%) at $5.7 \mathrm{Ma}$ and an increase during the early Pliocene. This pattern likely reflects higher biogenic productivity and/or reduced terrigenous deposition during the early Pliocene accompanied by a re-
F1. Bulk density vs. dry density, p. 6.

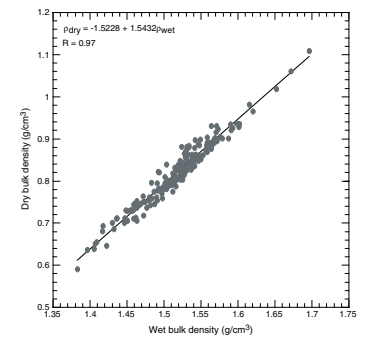

T1. Coarse-fraction grain-size analyses, p. 10.

T2. Biogenic silica content, p. 11.

T3. Edited MS measurements, p. 12.

T4. Edited GRA density and calculated dry bulk density, p. 13.

F2. Coarse-fraction size classes, p. 7.

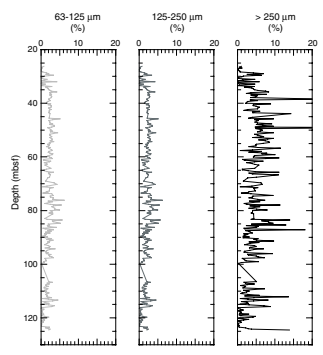

F3. MS, GRA density, coarse fraction, and biogenic silica, p. 8 .

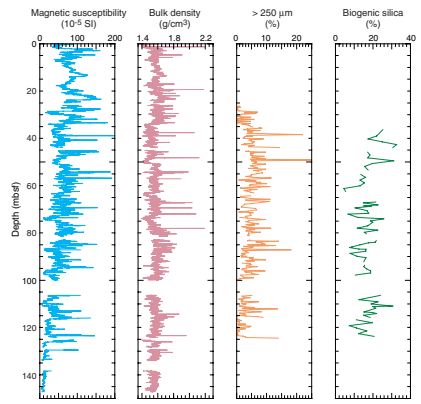




\section{J. GRÜTZNER}

Data Report: Core Data, Grain-Size Analysis, ANd Biogenic Silica

duced iceberg discharge in agreement with a climate warming during this time interval, as inferred by other studies (e.g., Abelmann et al., 1990; Hodell and Venz, 1992; Barker et al., 1999).

\section{ACKNOWLEDGMENTS}

I am grateful to the crew, technicians, and scientific staff of Leg 188. Julia Thiele and Marco Klann are thanked for technical assistance. A constructive review by Carol Pudsey improved the manuscript. This research used samples provided by the Ocean Drilling Program (ODP). The ODP is sponsored by the U.S. National Science Foundation (NSF) and participating countries under management of Joint Oceanographic Institutions (JOI), Inc. Funding for this research was provided by the Deutsche Forschungsgemeinschaft DFG, Project Number We 992/34.
T5. Statistics of measurements, p. 14.

T6. Correlation coefficients between sediment parameters, p. 15 .

F4. Biogenic silica and coarse fraction vs. age, p. 9.

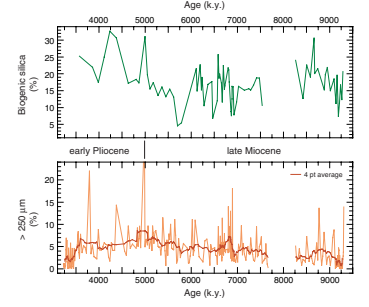

T7. Tie points used for depth to age conversion, p. 16. 


\section{REFERENCES}

Abelmann, A., Gersonde, R., and Spiess, V., 1990. Pliocene-Pleistocene paleoceanography in the Weddell Sea: siliceous microfossil evidence. In Bleil, U., and Thiede, J. (Eds.), Geologic History of the Polar Oceans: Arctic Versus Antarctic: NATO ASI Ser., Ser. C, 308:729-759.

Barker, P.F., Barrett, P.J., Cooper, A.K., and Huybrechts, P., 1999. Antarctic glacial history from numerical models and continental margin sediments. Palaeogeogr., Palaeoclimatol., Palaeoecol., 150:247-267.

Bonn, W.J., Gingele, F.X., Grobe, H., Mackensen, A., and Fütterer, D.K., 1998. Palaeoproductivity at the Antarctic continental margin: opal and barium records for the last 400 ka. Palaeogeogr., Palaeoclimat., Palaeoecol., 139:195-211.

Conley, D.J., 1998. An interlaboratory comparison for the measurement of biogenic silica in sediments. Mar. Chem., 63:39-48.

DeMaster, D.J., 1981. The supply and accumulation of silica in the marine environment. Geochim. Cosmochim. Acta, 45:1715-1732.

Hillenbrand, C.-D., and Fütterer, D.K., 2001. Neogene to Quaternary deposition of opal on the continental rise west of the Antarctic Peninsula, ODP Leg 178, Sites 1095, 1096, and 1101. In Barker, P.F., Camerlenghi, A., Acton, G.D., and Ramsay, A.T.S. (Eds.), Proc. ODP, Sci. Results, 178 [Online]. Available from World Wide Web: $<$ http://www-odp.tamu.edu/publications/178_SR/VOLUME/CHAPTERS/ SR178_23.PDF>. [Cited 2001-11-16]

Hodell, D.A., and Venz, K., 1992. Toward a high-resolution stable isotopic record of the Southern Ocean during the Pliocene-Pleistocene (4.8 to 0.8 Ma). In Kennett, J.P., Warnke, D.A. (Eds.), The Antarctic Paleoenvironment: A Perspective on Global Change (Pt. 1). Am. Geophys. Union, Antarct. Res. Ser., 56:265-310.

Müller, P.J., and Schneider, R., 1993. An automated leaching method for the determination of opal in sediments and particulate matter. Deep-Sea Res., 40:425-444.

O'Brien, P.E., Cooper, A.K., Richter, C., et al., 2001. Proc. ODP, Init. Repts., 188 [Online]. Available from World Wide Web: <http://www-odp.tamu.edu/publications/188_IR/188ir.htm>. [Cited 2001-04-05].

Shipboard Scientific Party, 2001. Site 1165. In O'Brien, P.E., Cooper, A.K., Richter, C., et al., Proc. ODP, Init. Repts., 188, 1-191 [Online]. Available from World Wide Web: $<$ http://www-odp.tamu.edu/publications/188_IR/VOLUME/CHAPTERS/

IR188_03.PDF>. [Cited 2001-04-05] 


\section{J. GRÜTZNER}

Data Report: Core Data, Grain-Size Analysis, ANd Biogenic Silica

Figure F1. Bulk density vs. dry density derived from moisture and density measurements (MAD) made on discrete samples during Leg 188.

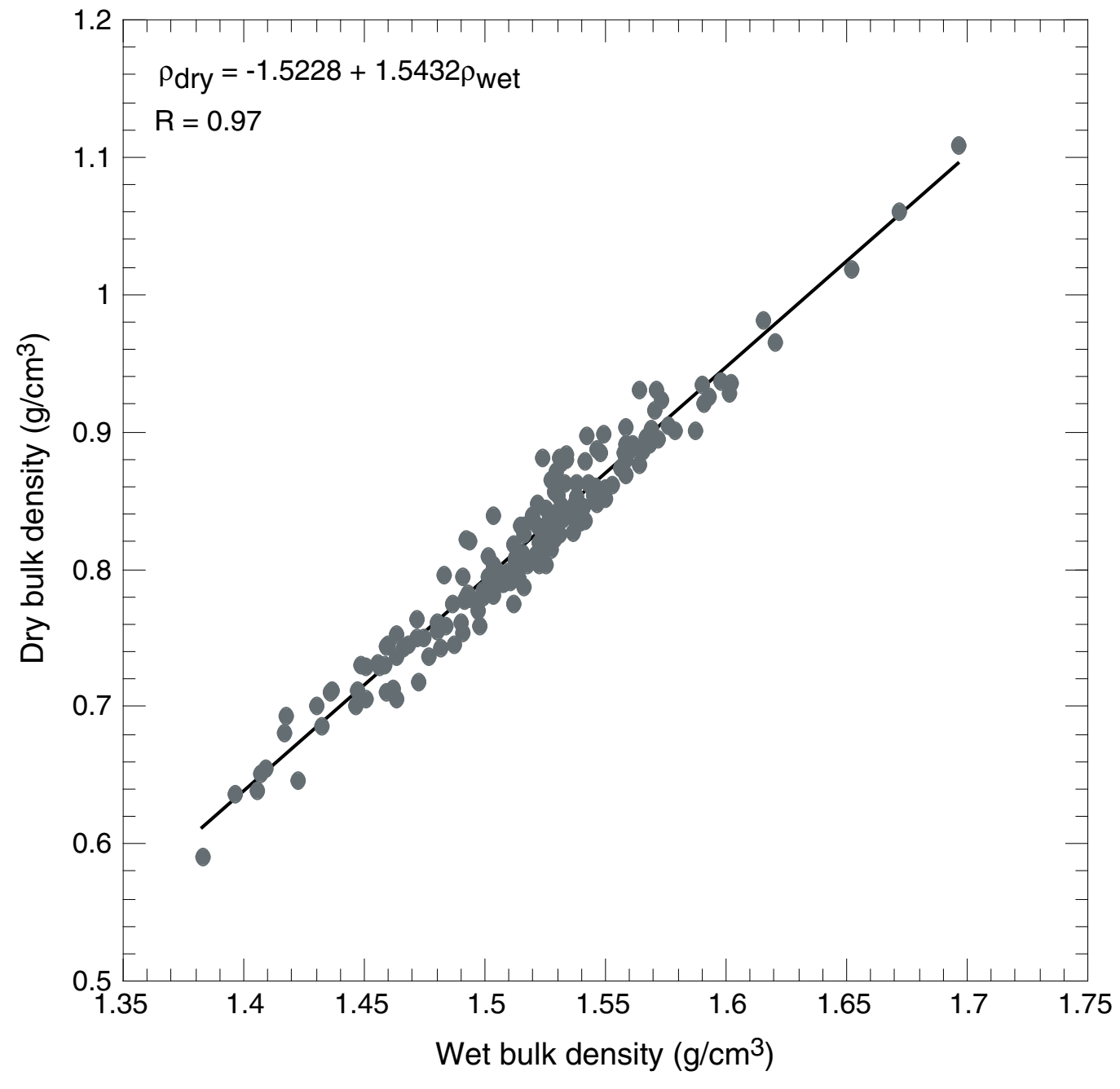




\section{J. GRÜTZNER}

Data Report: Core Data, Grain-Size Analysis, and Biogenic Silica

Figure F2. Weight percentages of coarse-fraction size classes $(63-125,125-250$, and $>250 \mu \mathrm{m})$ vs. depth.

$63-125 \mu \mathrm{m}$

(\%)

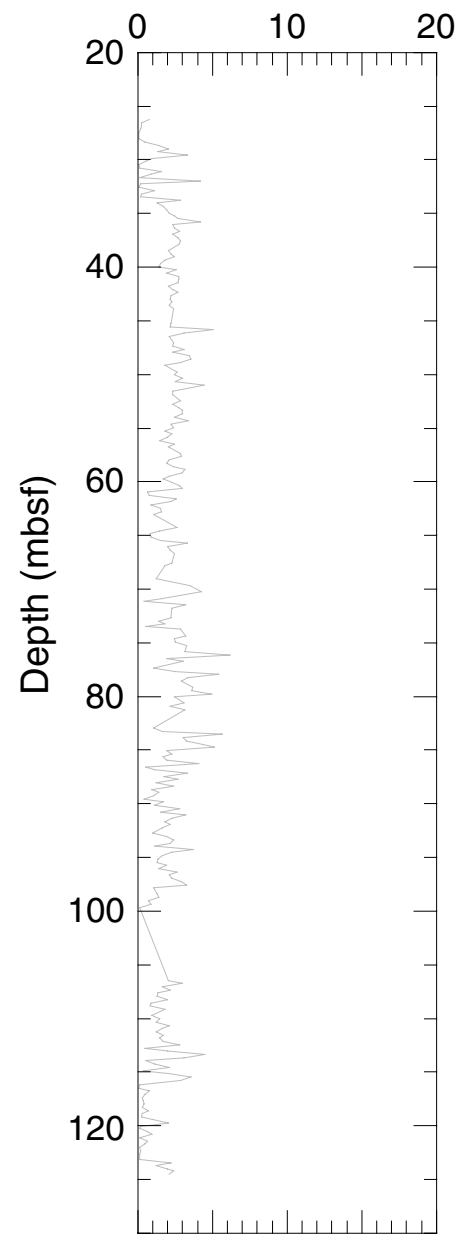

$125-250 \mu \mathrm{m}$

(\%)

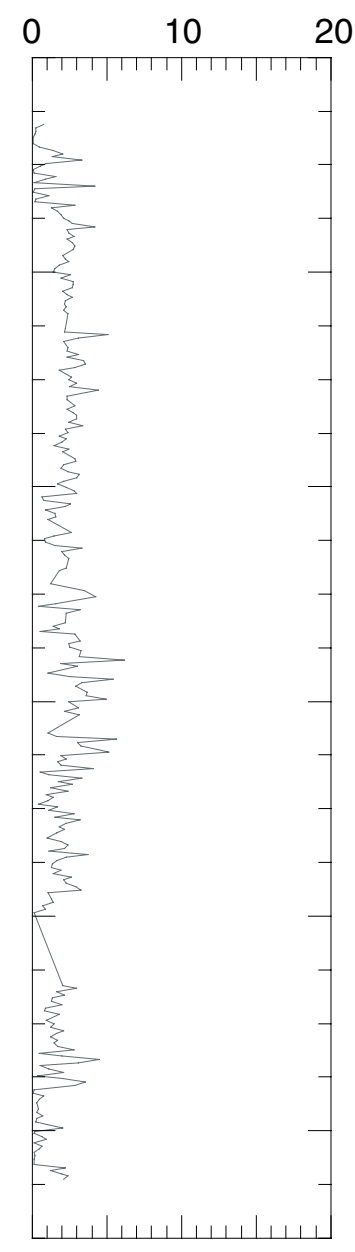

$>250 \mu \mathrm{m}$

(\%)

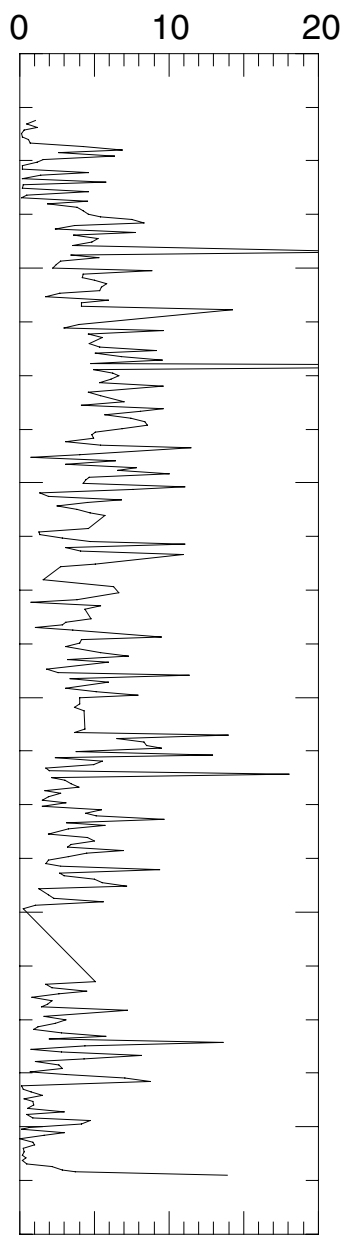




\section{J. GRÜTZNER}

Data Report: Core Data, Grain-Size Analysis, And Biogenic Silica

Figure F3. Magnetic susceptibility, GRA bulk density, weight percentage of $>250-\mu \mathrm{m}$ size fraction, and biogenic silica content vs. depth.

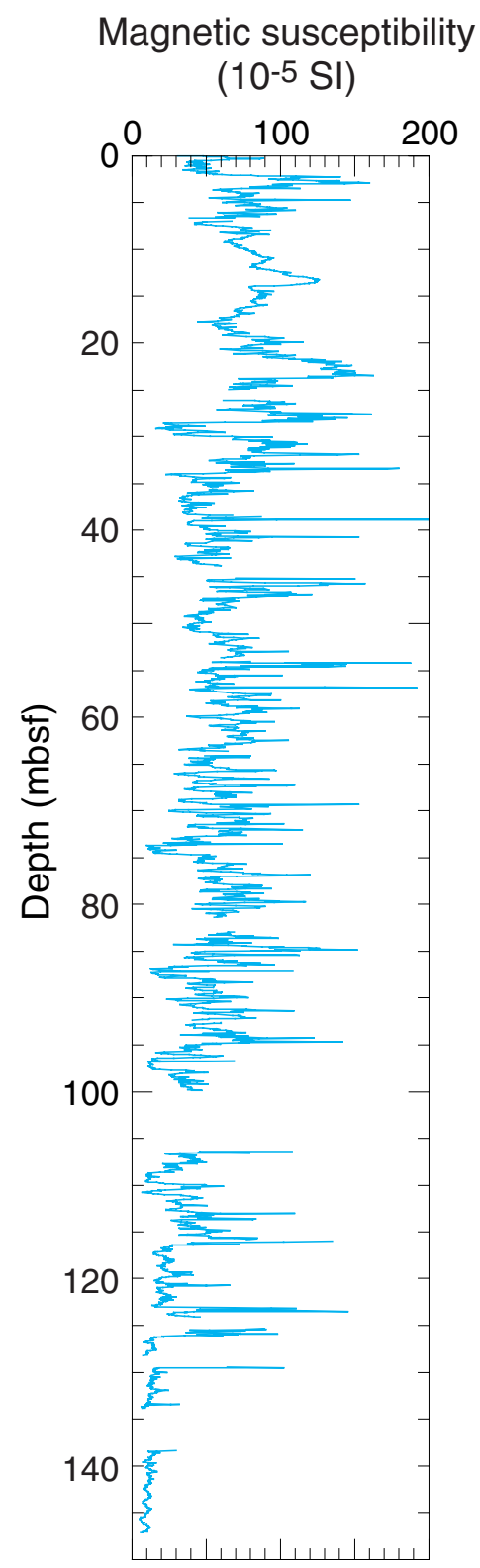

$$
\text { Bulk density }
$$
$\left(\mathrm{g} / \mathrm{cm}^{3}\right)$

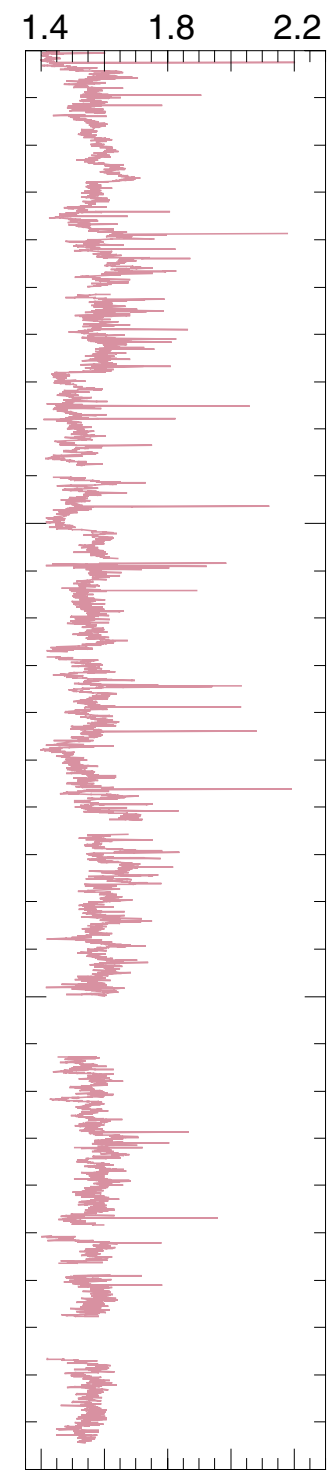

$>250 \mu \mathrm{m}$

(\%)

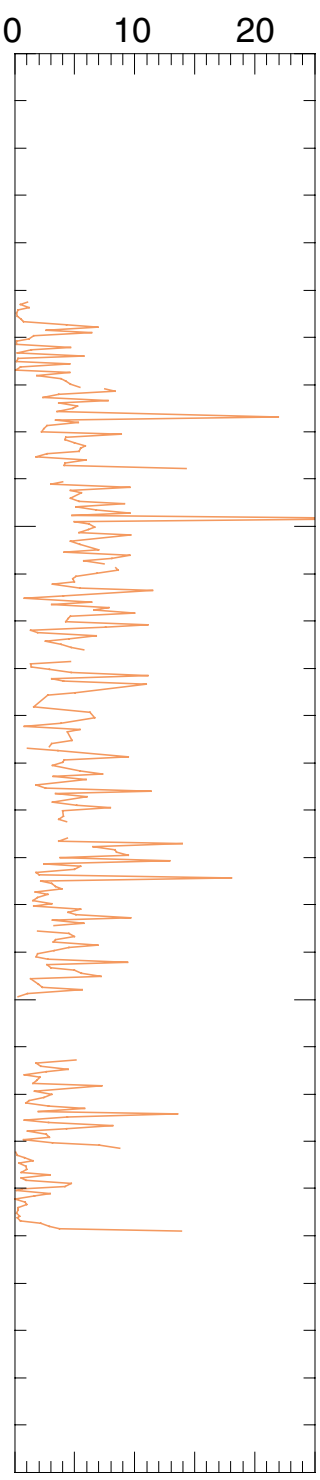

Biogenic silica

(\%)

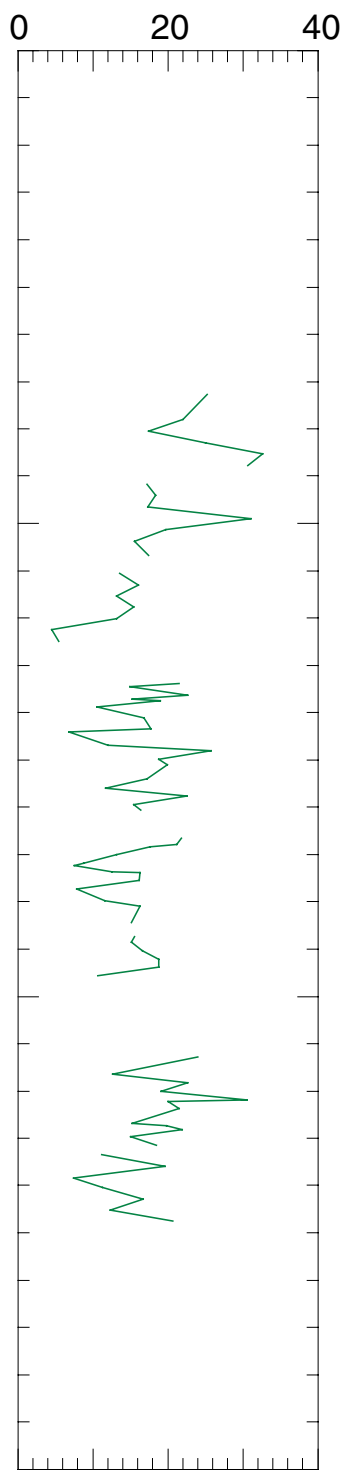




\section{J. GRÜTZNER}

Data Report: Core Data, Grain-Size Analysis, ANd Biogenic Silica

Figure F4. Biogenic silica content and weight percentage of $>250-\mu \mathrm{m}$ size fraction vs. age. Depth to age conversion is according to Table T7, p. 16.
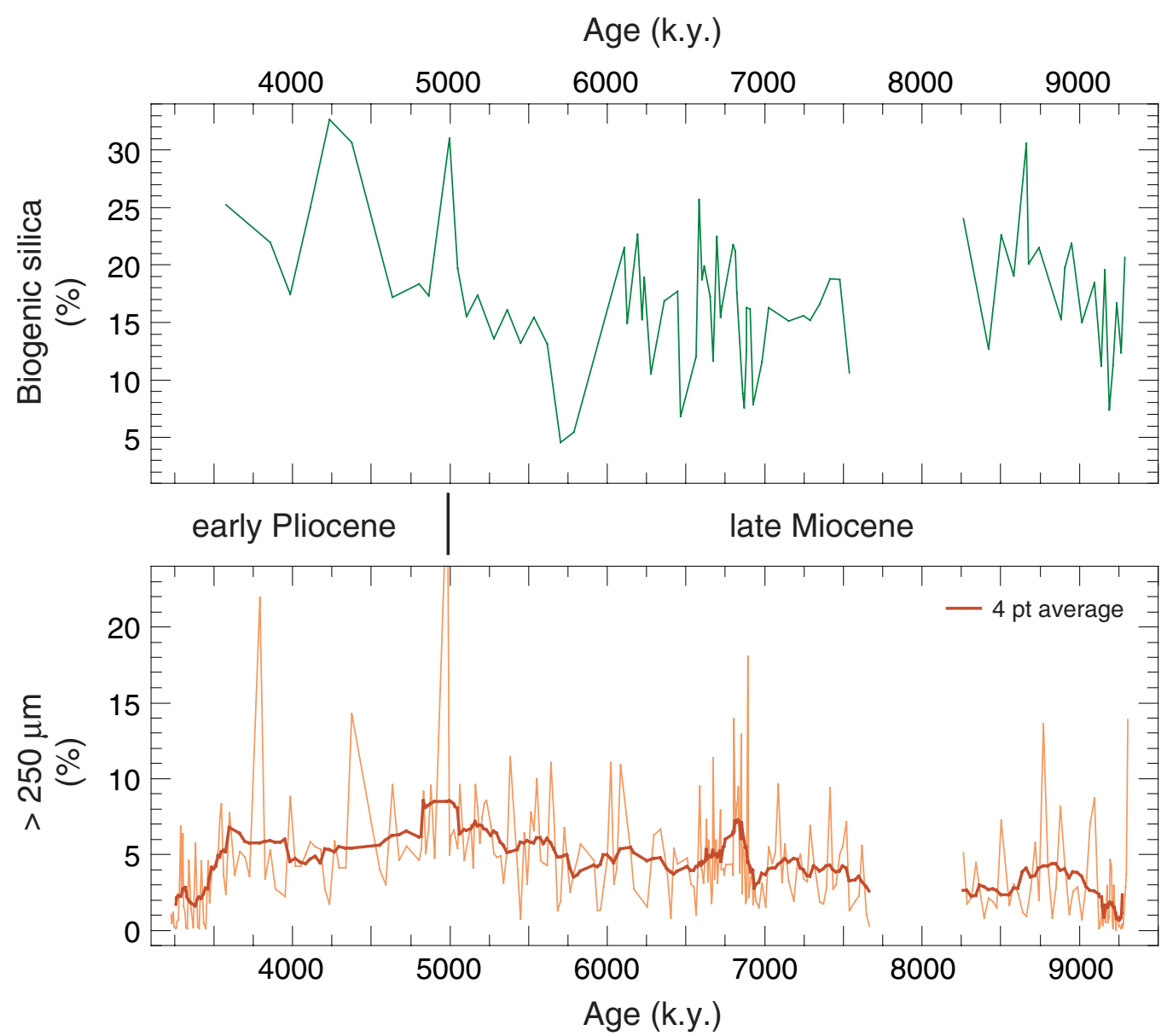
J. GRÜTZNER

Data Report: Core Data, Grain-Size AnAlysis, ANd Biogenic Silica

Table T1. Results of coarse fraction grain size analyses, Site 1165.

\begin{tabular}{|c|c|c|c|c|c|c|}
\hline $\begin{array}{l}\text { Core, section, } \\
\text { interval }(\mathrm{cm})\end{array}$ & $\begin{array}{l}\text { Depth } \\
\text { (mbsf) }\end{array}$ & $\begin{array}{c}>63 \mu \mathrm{m} \\
(\%)\end{array}$ & $\begin{array}{c}>125 \mu \mathrm{m} \\
(\%)\end{array}$ & $\begin{array}{c}>250 \mu \mathrm{m} \\
(\%)\end{array}$ & $\begin{array}{c}63-125 \mu \mathrm{m} \\
(\%)\end{array}$ & $\begin{array}{c}125-250 \mu \mathrm{m} \\
(\%)\end{array}$ \\
\hline \multicolumn{7}{|l|}{ 188-1165B- } \\
\hline $4 \mathrm{H}-1,45-47$ & 26.25 & 2.56 & 1.78 & 1.04 & 0.78 & 0.74 \\
\hline $4 \mathrm{H}-1,75-77$ & 26.55 & 0.88 & 0.66 & 0.46 & 0.22 & 0.21 \\
\hline $4 \mathrm{H}-1,105-107$ & 26.85 & 2.00 & 1.76 & 1.18 & 0.24 & 0.58 \\
\hline $4 \mathrm{H}-1,135-137$ & 27.15 & 0.61 & 0.45 & 0.29 & 0.16 & 0.16 \\
\hline $4 \mathrm{H}-2,15-17$ & 27.45 & 0.33 & 0.23 & 0.12 & 0.10 & 0.11 \\
\hline $4 \mathrm{H}-2,45-47$ & 27.75 & 0.38 & 0.29 & 0.18 & 0.10 & 0.10 \\
\hline $4 \mathrm{H}-2,75-77$ & 28.05 & 0.62 & 0.59 & 0.57 & 0.04 & 0.02 \\
\hline $4 \mathrm{H}-2,105-107$ & 28.35 & 1.71 & 1.23 & 0.70 & 0.48 & 0.53 \\
\hline $4 \mathrm{H}-2,135-137$ & 28.65 & 7.08 & 5.68 & 4.28 & 1.40 & 1.40 \\
\hline $4 \mathrm{H}-3,15-17$ & 28.95 & 10.89 & 8.87 & 6.89 & 2.02 & 1.99 \\
\hline $4 \mathrm{H}-3,45-47$ & 29.25 & 5.20 & 3.88 & 2.60 & 1.31 & 1.29 \\
\hline $4 \mathrm{H}-3,75-77$ & 29.55 & 12.63 & 9.33 & 6.37 & 3.30 & 2.97 \\
\hline $4 \mathrm{H}-3,105-107$ & 29.85 & 3.18 & 2.26 & 1.58 & 0.92 & 0.68 \\
\hline $4 \mathrm{H}-3,135-137$ & 30.15 & 2.29 & 1.74 & 1.16 & 0.55 & 0.58 \\
\hline $4 \mathrm{H}-4,15-17$ & 30.45 & 0.33 & 0.25 & 0.16 & 0.08 & 0.09 \\
\hline $4 \mathrm{H}-4,45-47$ & 30.75 & 0.37 & 0.25 & 0.15 & 0.12 & 0.10 \\
\hline $4 \mathrm{H}-4,75-77$ & 31.05 & 7.94 & 6.37 & 4.62 & 1.56 & 1.76 \\
\hline $4 \mathrm{H}-4,105-107$ & 31.35 & 3.08 & 2.23 & 1.39 & 0.85 & 0.83 \\
\hline $4 \mathrm{H}-4,135-137$ & 31.65 & 0.45 & 0.32 & 0.19 & 0.13 & 0.13 \\
\hline $4 \mathrm{H}-5,15-17$ & 31.95 & 13.53 & 9.31 & 5.75 & 4.22 & 3.57 \\
\hline $4 \mathrm{H}-5,45-47$ & 32.25 & 0.55 & 0.40 & 0.25 & 0.15 & 0.15 \\
\hline $4 \mathrm{H}-5,75-77$ & 32.55 & 0.27 & 0.21 & 0.15 & 0.07 & 0.06 \\
\hline $4 \mathrm{H}-5,105-107$ & 32.85 & 6.81 & 5.72 & 4.59 & 1.09 & 1.13 \\
\hline $4 \mathrm{H}-5,135-137$ & 33.15 & 0.94 & 0.71 & 0.46 & 0.23 & 0.25 \\
\hline $4 \mathrm{H}-6,15-17$ & 33.45 & 0.38 & 0.22 & 0.10 & 0.16 & 0.12 \\
\hline $4 \mathrm{H}-6,45-47$ & 33.75 & 9.78 & 6.92 & 4.57 & 2.86 & 2.36 \\
\hline $4 \mathrm{H}-6,75-77$ & 34.05 & 4.33 & 3.04 & 1.84 & 1.29 & 1.19 \\
\hline $4 \mathrm{H}-6,105-107$ & 34.35 & 7.67 & 5.95 & 3.84 & 1.72 & 2.11 \\
\hline $4 \mathrm{H}-6,135-137$ & 34.65 & 7.89 & 5.93 & 4.28 & 1.95 & 1.66 \\
\hline $4 \mathrm{H}-7,15-17$ & 34.95 & 8.36 & 6.26 & 4.60 & 2.11 & 1.65 \\
\hline $4 \mathrm{H}-7,45-47$ & 35.25 & 9.95 & 7.48 & 5.43 & 2.47 & 2.05 \\
\hline $5 \mathrm{H}-1,15-17$ & 35.45 & 13.47 & 10.76 & 7.50 & 2.71 & 3.27 \\
\hline $5 \mathrm{H}-1,45-47$ & 35.75 & 16.40 & 12.19 & 8.32 & 4.21 & 3.87 \\
\hline $5 \mathrm{H}-1,75-77$ & 36.05 & 7.76 & 5.41 & 3.67 & 2.36 & 1.74 \\
\hline $5 \mathrm{H}-1,105-107$ & 36.35 & 6.42 & 3.99 & 2.40 & 2.44 & 1.59 \\
\hline $5 \mathrm{H}-1,135-137$ & 36.65 & 12.80 & 10.02 & 7.77 & 2.78 & 2.25 \\
\hline $5 \mathrm{H}-2,15-17$ & 36.95 & 7.93 & 5.61 & 3.62 & 2.32 & 1.98 \\
\hline $5 \mathrm{H}-2,45-47$ & 37.25 & 9.81 & 7.13 & 5.22 & 2.69 & 1.91 \\
\hline $5 \mathrm{H}-2,75-77$ & 37.55 & 10.07 & 7.19 & 4.81 & 2.87 & 2.38 \\
\hline $5 \mathrm{H}-2,105-107$ & 37.85 & 8.10 & 5.37 & 3.55 & 2.73 & 1.82 \\
\hline $5 \mathrm{H}-3,15-17$ & 38.45 & 25.83 & 23.81 & 21.97 & 2.02 & 1.84 \\
\hline $5 \mathrm{H}-3,45-47$ & 38.75 & 7.43 & 5.23 & 3.41 & 2.20 & 1.83 \\
\hline $5 \mathrm{H}-3,75-77$ & 39.05 & 9.64 & 7.20 & 5.29 & 2.44 & 1.92 \\
\hline $5 \mathrm{H}-3,105-107$ & 39.35 & 5.88 & 4.09 & 2.72 & 1.78 & 1.38 \\
\hline $5 \mathrm{H}-3,135-137$ & 39.65 & 5.41 & 3.87 & 2.46 & 1.54 & 1.41 \\
\hline $5 \mathrm{H}-4,15-17$ & 39.95 & 4.93 & 3.52 & 2.21 & 1.41 & 1.31 \\
\hline $5 \mathrm{H}-4,45-47$ & 40.25 & 13.86 & 11.28 & 8.84 & 2.59 & 2.43 \\
\hline $5 \mathrm{H}-4,75-77$ & 40.55 & 7.90 & 5.95 & 4.24 & 1.95 & 1.71 \\
\hline $5 \mathrm{H}-4,105-107$ & 40.85 & 9.15 & 6.41 & 4.21 & 2.74 & 2.20 \\
\hline $5 \mathrm{H}-4,135-137$ & 41.15 & 10.51 & 7.79 & 4.99 & 2.72 & 2.80 \\
\hline $5 \mathrm{H}-5,15-17$ & 41.45 & 10.66 & 7.99 & 5.85 & 2.66 & 2.14 \\
\hline $5 \mathrm{H}-5,45-47$ & 41.75 & 9.39 & 7.36 & 5.49 & 2.03 & 1.87 \\
\hline $5 \mathrm{H}-5,75-77$ & 42.05 & 9.72 & 7.41 & 5.33 & 2.31 & 2.07 \\
\hline $5 \mathrm{H}-5,105-107$ & 42.35 & 7.21 & 4.55 & 2.69 & 2.66 & 1.86 \\
\hline $5 \mathrm{H}-5,135-137$ & 42.65 & 5.28 & 3.05 & 1.75 & 2.23 & 1.30 \\
\hline $5 \mathrm{H}-6,15-17$ & 42.95 & 9.67 & 7.54 & 5.92 & 2.13 & 1.63 \\
\hline $5 \mathrm{H}-6,45-47$ & 43.25 & 8.57 & 6.26 & 4.14 & 2.31 & 2.12 \\
\hline $5 \mathrm{H}-6,75-77$ & 43.55 & 7.84 & 5.73 & 4.13 & 2.11 & 1.60 \\
\hline $5 \mathrm{H}-6,105-107$ & 43.85 & 19.02 & 16.63 & 14.27 & 2.39 & 2.36 \\
\hline $6 \mathrm{H}-1,45-47$ & 45.25 & 8.34 & 6.14 & 3.97 & 2.20 & 2.17 \\
\hline $6 \mathrm{H}-1,75-77$ & 45.55 & 7.27 & 5.10 & 3.01 & 2.17 & 2.09 \\
\hline $6 \mathrm{H}-1,105-107$ & 45.85 & 19.46 & 14.42 & 9.60 & 5.04 & 4.82 \\
\hline $6 \mathrm{H}-1,135-137$ & 46.15 & 10.51 & 7.41 & 4.62 & 3.09 & 2.79 \\
\hline $6 \mathrm{H}-2,15-17$ & 46.45 & 9.35 & 7.26 & 5.54 & 2.10 & 1.71 \\
\hline $6 \mathrm{H}-2,75-77$ & 47.05 & 9.33 & 6.96 & 4.64 & 2.38 & 2.32 \\
\hline
\end{tabular}

Note: Only a portion of this table appears here. The complete table is available in ASCII. 


\section{J. GRÜTZNER}

Data Report: Core Data, Grain-Size Analysis, AND Biogenic Silica

Table T2. Biogenic silica content, Site 1165.

\begin{tabular}{ccc}
\hline $\begin{array}{c}\text { Core, section, } \\
\text { interval (cm) }\end{array}$ & $\begin{array}{c}\text { Depth } \\
\text { (mbsf) }\end{array}$ & $\begin{array}{c}\text { Biogenic } \\
\text { silica } \\
(\%)\end{array}$ \\
\hline $188-1165 \mathrm{~B}-$ & & \\
$5 \mathrm{H}-1,105-107$ & 36.35 & 25.27 \\
$5 \mathrm{H}-3,75-77$ & 39.05 & 21.96 \\
$5 \mathrm{H}-4,45-47$ & 40.25 & 17.43 \\
$5 \mathrm{H}-5,15-17$ & 41.45 & 25.01 \\
$5 \mathrm{H}-5,135-137$ & 42.65 & 32.66 \\
$5 \mathrm{H}-6,107-107$ & 43.85 & 30.65 \\
$6 \mathrm{H}-1,107-107$ & 45.85 & 17.18 \\
$6 \mathrm{H}-2,75-77$ & 47.05 & 18.33 \\
$6 \mathrm{H}-3,45-47$ & 48.25 & 17.35 \\
$6 \mathrm{H}-4,15-17$ & 49.45 & 31.03 \\
$6 \mathrm{H}-4,135-137$ & 50.65 & 19.72 \\
$6 \mathrm{H}-5,105-107$ & 51.85 & 15.54 \\
$6 \mathrm{H}-6,105-107$ & 53.35 & 17.39 \\
$188-1165 \mathrm{C}-$ & & \\
$1 \mathrm{R}-1,126-128$ & 55.27 & 13.61 \\
$1 \mathrm{R}-2,97-99$ & 56.48 & 16.08 \\
$1 \mathrm{R}-3,63-65$ & 57.64 & 13.18 \\
$1 \mathrm{R}-4,36-38$ & 58.87 & 15.42 \\
$1 \mathrm{R}-5,6-8$ & 60.07 & 13.11 \\
$1 \mathrm{R}-5,126-128$ & 61.27 & 4.52 \\
$1 \mathrm{R}-6,96-98$ & 62.47 & 5.49 \\
$188-1165 \mathrm{~B}-$ & & \\
$8 \mathrm{H}-3,15-17$ & 66.95 & 21.48 \\
$8 \mathrm{H}-3,45-47$ & 67.25 & 14.94 \\
$8 \mathrm{H}-3,135-137$ & 68.15 & 22.64 \\
$8 \mathrm{H}-4,30-32$ & 68.60 & 15.27 \\
$8 \mathrm{H}-4,45-47$ & 68.75 & 18.94 \\
$8 \mathrm{H}-4,105-107$ & 69.35 & 10.57 \\
$8 \mathrm{H}-5,75-77$ & 70.55 & 16.85 \\
$8 \mathrm{H}-6,45-47$ & 71.75 & 17.70 \\
$8 \mathrm{H}-6,75-77$ & 72.05 & 6.81 \\
$9 \mathrm{H}-1,15-17$ & 73.45 & 12.03 \\
$9 \mathrm{H}-1,75-77$ & 74.05 & 25.71 \\
$9 \mathrm{H}-2,15-17$ & 74.95 & 18.77 \\
$9 \mathrm{H}-2,75-77$ & 75.55 & 19.89 \\
$9 \mathrm{H}-3,75-77$ & 77.05 & 17.26 \\
$9 \mathrm{H}-4,15-17$ & 77.95 & 11.68 \\
$9 \mathrm{H}-4,105-107$ & 78.85 & 22.48 \\
$9 \mathrm{H}-5,45-47$ & 79.75 & 15.43 \\
& & \\
& &
\end{tabular}

\begin{tabular}{lrr}
\hline $\begin{array}{c}\text { Core, section, } \\
\text { interval (cm) }\end{array}$ & $\begin{array}{r}\text { Depth } \\
\text { (mbsf) }\end{array}$ & $\begin{array}{c}\text { Biogenic } \\
\text { silica } \\
(\%)\end{array}$ \\
\hline $9 \mathrm{H}-5,105-107$ & 80.35 & 16.42 \\
$10 \mathrm{H}-1,45-47$ & 83.25 & 21.78 \\
$10 \mathrm{H}-1,110-112$ & 83.90 & 21.18 \\
$10 \mathrm{H}-1,135-137$ & 84.15 & 17.60 \\
$10 \mathrm{H}-2,75-77$ & 85.05 & 13.18 \\
$10 \mathrm{H}-3,15-17$ & 85.95 & 8.81 \\
$10 \mathrm{H}-3,34-36$ & 86.14 & 7.56 \\
$10 \mathrm{H}-3,105-107$ & 86.85 & 12.56 \\
$10 \mathrm{H}-3,110-112$ & 86.90 & 16.26 \\
$10 \mathrm{H}-4,45-47$ & 87.75 & 16.18 \\
$10 \mathrm{H}-4,135-137$ & 88.65 & 7.84 \\
$10 \mathrm{H}-5,105-107$ & 89.85 & 11.58 \\
$10 \mathrm{H}-6,15-17$ & 90.45 & 16.26 \\
$10 \mathrm{H}-7,45-47$ & 92.25 & 15.10 \\
$11 \mathrm{H}-1,135-137$ & 93.65 & 15.58 \\
$11 \mathrm{H}-2,45-47$ & 94.25 & 15.17 \\
$11 \mathrm{H}-2,135-137$ & 95.15 & 16.59 \\
$11 \mathrm{H}-3,75-77$ & 96.05 & 18.79 \\
$11 \mathrm{H}-4,15-17$ & 96.95 & 18.74 \\
$11 \mathrm{H}-4,105-107$ & 97.85 & 10.63 \\
$13 \mathrm{H}-1,15-17$ & 106.45 & 24.02 \\
$13 \mathrm{H}-2,45-47$ & 108.25 & 12.64 \\
$13 \mathrm{H}-2,135-137$ & 109.15 & 22.62 \\
$13 \mathrm{H}-3,75-77$ & 110.05 & 19.08 \\
$13 \mathrm{H}-4,15-17$ & 110.95 & 30.59 \\
$13 \mathrm{H}-4,30-32$ & 111.10 & 20.08 \\
$13 \mathrm{H}-4,105-107$ & 111.85 & 21.50 \\
$13 \mathrm{H}-5,111-113$ & 113.41 & 15.27 \\
$13 \mathrm{H}-5,135-137$ & 113.65 & 19.78 \\
$13 \mathrm{H}-6,31-33$ & 114.11 & 21.91 \\
$13 \mathrm{H}-6,105-107$ & 114.85 & 15.00 \\
$13 \mathrm{H}-7,45-47$ & 115.75 & 18.44 \\
$14 \mathrm{H}-1,96-98$ & 116.77 & 11.20 \\
$14 \mathrm{H}-2,65-67$ & 117.96 & 19.59 \\
$14 \mathrm{H}-3,36-38$ & 119.17 & 7.42 \\
$14 \mathrm{H}-4,6-8$ & 120.22 & 11.23 \\
$14 \mathrm{H}-4,126-128$ & 121.42 & 16.65 \\
$14 \mathrm{H}-5,96-98$ & 122.62 & 12.35 \\
$14 \mathrm{H}-6,65-67$ & 123.72 & 20.69 \\
\hline & & \\
\hline
\end{tabular}

Note: This table is also available in ASCII. 
Table T3. Edited magnetic susceptibility measurements, Site 1165.

\begin{tabular}{|c|c|c|}
\hline $\begin{array}{l}\text { Core, section, } \\
\text { interval }(\mathrm{cm})\end{array}$ & $\begin{array}{l}\text { Depth } \\
\text { (mbsf) }\end{array}$ & $\begin{array}{c}\text { Magnetic } \\
\text { susceptibility } \\
\left(10^{-5} \mathrm{SI}\right)\end{array}$ \\
\hline \multicolumn{3}{|l|}{ 188-1165B- } \\
\hline $1 \mathrm{H}-1,2$ & 0.02 & 30.71 \\
\hline $1 \mathrm{H}-1,4$ & 0.04 & 42.02 \\
\hline $1 \mathrm{H}-1,6$ & 0.06 & 47.72 \\
\hline $1 \mathrm{H}-1,8$ & 0.08 & 50.73 \\
\hline $1 \mathrm{H}-1,10$ & 0.10 & 57.74 \\
\hline $1 \mathrm{H}-1,12$ & 0.12 & 59.74 \\
\hline $1 \mathrm{H}-1,14$ & 0.14 & 62.05 \\
\hline $1 \mathrm{H}-1,16$ & 0.16 & 63.75 \\
\hline $1 \mathrm{H}-1,18$ & 0.18 & 63.06 \\
\hline $1 \mathrm{H}-1,20$ & 0.20 & 63.06 \\
\hline $1 \mathrm{H}-1,22$ & 0.22 & 64.07 \\
\hline $1 \mathrm{H}-1,24$ & 0.24 & 65.07 \\
\hline $1 \mathrm{H}-1,26$ & 0.26 & 70.38 \\
\hline $1 \mathrm{H}-1,28$ & 0.28 & 88.78 \\
\hline $1 \mathrm{H}-1,30$ & 0.30 & 86.09 \\
\hline $1 \mathrm{H}-1,32$ & 0.32 & 73.80 \\
\hline $1 \mathrm{H}-1,34$ & 0.34 & 70.80 \\
\hline $1 \mathrm{H}-1,36$ & 0.36 & 67.81 \\
\hline $1 \mathrm{H}-1,38$ & 0.38 & 67.11 \\
\hline $1 \mathrm{H}-1,40$ & 0.40 & 64.12 \\
\hline $1 \mathrm{H}-1,42$ & 0.42 & 65.42 \\
\hline $1 \mathrm{H}-1,44$ & 0.44 & 65.13 \\
\hline $1 \mathrm{H}-1,46$ & 0.46 & 60.43 \\
\hline $1 \mathrm{H}-1,48$ & 0.48 & 53.44 \\
\hline $1 \mathrm{H}-1,50$ & 0.50 & 48.14 \\
\hline $1 \mathrm{H}-1,52$ & 0.52 & 43.46 \\
\hline $1 \mathrm{H}-1,54$ & 0.54 & 42.47 \\
\hline $1 \mathrm{H}-1,56$ & 0.56 & 46.18 \\
\hline $1 \mathrm{H}-1,58$ & 0.58 & 47.19 \\
\hline $1 \mathrm{H}-1,60$ & 0.60 & 42.21 \\
\hline $1 \mathrm{H}-1,62$ & 0.62 & 39.22 \\
\hline $1 \mathrm{H}-1,64$ & 0.64 & 36.93 \\
\hline $1 \mathrm{H}-1,66$ & 0.66 & 37.54 \\
\hline $1 \mathrm{H}-1,68$ & 0.68 & 39.55 \\
\hline $1 \mathrm{H}-1,70$ & 0.70 & 40.97 \\
\hline $1 \mathrm{H}-1,72$ & 0.72 & 39.28 \\
\hline $1 \mathrm{H}-1,74$ & 0.74 & 38.29 \\
\hline $1 \mathrm{H}-1,76$ & 0.76 & 39.00 \\
\hline $1 \mathrm{H}-1,78$ & 0.78 & 44.01 \\
\hline $1 \mathrm{H}-1,80$ & 0.80 & 48.33 \\
\hline $1 \mathrm{H}-1,82$ & 0.82 & 45.04 \\
\hline $1 \mathrm{H}-1,84$ & 0.84 & 41.65 \\
\hline $1 \mathrm{H}-1,86$ & 0.86 & 44.06 \\
\hline $1 \mathrm{H}-1,88$ & 0.88 & 49.38 \\
\hline $1 \mathrm{H}-1,90$ & 0.90 & 50.09 \\
\hline $1 \mathrm{H}-1,92$ & 0.92 & 49.10 \\
\hline $1 \mathrm{H}-1,94$ & 0.94 & 52.41 \\
\hline $1 \mathrm{H}-1,96$ & 0.96 & 46.72 \\
\hline $1 \mathrm{H}-1,98$ & 0.98 & 43.44 \\
\hline $1 \mathrm{H}-1,100$ & 1.00 & 40.45 \\
\hline $1 \mathrm{H}-1,102$ & 1.02 & 38.47 \\
\hline $1 \mathrm{H}-1,104$ & 1.04 & 39.78 \\
\hline $1 \mathrm{H}-1,106$ & 1.06 & 38.49 \\
\hline $1 \mathrm{H}-1,108$ & 1.08 & 36.21 \\
\hline $1 \mathrm{H}-1,110$ & 1.10 & 38.22 \\
\hline $1 \mathrm{H}-1,112$ & 1.12 & 42.24 \\
\hline $1 \mathrm{H}-1,114$ & 1.14 & 44.25 \\
\hline $1 \mathrm{H}-1,116$ & 1.16 & 44.57 \\
\hline $1 \mathrm{H}-1,118$ & 1.18 & 43.28 \\
\hline $1 \mathrm{H}-1,120$ & 1.20 & 49.29 \\
\hline $1 \mathrm{H}-1,122$ & 1.22 & 47.62 \\
\hline $1 \mathrm{H}-1,124$ & 1.24 & 44.93 \\
\hline $1 \mathrm{H}-1,126$ & 1.26 & 43.94 \\
\hline $1 \mathrm{H}-1,128$ & 1.28 & 43.95 \\
\hline $1 \mathrm{H}-1,130$ & 1.30 & 47.97 \\
\hline $1 \mathrm{H}-1,132$ & 1.32 & 49.69 \\
\hline $1 \mathrm{H}-1,134$ & 1.34 & 51.00 \\
\hline
\end{tabular}

\begin{tabular}{|c|c|c|}
\hline $\begin{array}{l}\text { Core, section, } \\
\text { interval }(\mathrm{cm})\end{array}$ & $\begin{array}{l}\text { Depth } \\
\text { (mbsf) }\end{array}$ & $\begin{array}{c}\text { Magnetic } \\
\text { susceptibility } \\
\left(10^{-5} \mathrm{SI}\right)\end{array}$ \\
\hline $1 \mathrm{H}-1,136$ & 1.36 & 53.41 \\
\hline $1 \mathrm{H}-1,138$ & 1.38 & 52.73 \\
\hline $1 \mathrm{H}-1,140$ & 1.40 & 49.74 \\
\hline $1 \mathrm{H}-1,142$ & 1.42 & 45.76 \\
\hline $1 \mathrm{H}-1,144$ & 1.44 & 45.08 \\
\hline $1 \mathrm{H}-1,146$ & 1.46 & 42.09 \\
\hline $1 \mathrm{H}-1,148$ & 1.48 & 37.10 \\
\hline $1 \mathrm{H}-2,2$ & 1.52 & 34.31 \\
\hline $1 \mathrm{H}-2,4$ & 1.54 & 38.31 \\
\hline $1 \mathrm{H}-2,6$ & 1.56 & 39.71 \\
\hline $1 \mathrm{H}-2,8$ & 1.58 & 40.32 \\
\hline $1 \mathrm{H}-2,10$ & 1.60 & 41.32 \\
\hline $1 \mathrm{H}-2,12$ & 1.62 & 44.32 \\
\hline $1 \mathrm{H}-2,14$ & 1.64 & 46.03 \\
\hline $1 \mathrm{H}-2,16$ & 1.66 & 50.03 \\
\hline $1 \mathrm{H}-2,18$ & 1.68 & 53.33 \\
\hline $1 \mathrm{H}-2,20$ & 1.70 & 58.73 \\
\hline $1 \mathrm{H}-2,22$ & 1.72 & 56.04 \\
\hline $1 \mathrm{H}-2,24$ & 1.74 & 52.34 \\
\hline $1 \mathrm{H}-2,26$ & 1.76 & 48.34 \\
\hline $1 \mathrm{H}-2,28$ & 1.78 & 47.35 \\
\hline $1 \mathrm{H}-2,30$ & 1.80 & 45.05 \\
\hline $1 \mathrm{H}-2,32$ & 1.82 & 44.05 \\
\hline $1 \mathrm{H}-2,34$ & 1.84 & 45.35 \\
\hline $1 \mathrm{H}-2,36$ & 1.86 & 49.36 \\
\hline $1 \mathrm{H}-2,38$ & 1.88 & 52.36 \\
\hline $1 \mathrm{H}-2,40$ & 1.90 & 55.36 \\
\hline $1 \mathrm{H}-2,42$ & 1.92 & 57.07 \\
\hline $1 \mathrm{H}-2,44$ & 1.94 & 55.77 \\
\hline $1 \mathrm{H}-2,46$ & 1.96 & 54.77 \\
\hline $1 \mathrm{H}-2,48$ & 1.98 & 54.77 \\
\hline $1 \mathrm{H}-2,50$ & 2.00 & 57.08 \\
\hline $1 \mathrm{H}-2,52$ & 2.02 & 59.08 \\
\hline $1 \mathrm{H}-2,54$ & 2.04 & 60.79 \\
\hline $1 \mathrm{H}-2,56$ & 2.06 & 66.80 \\
\hline $1 \mathrm{H}-2,58$ & 2.08 & 72.80 \\
\hline $1 \mathrm{H}-2,60$ & 2.10 & 76.11 \\
\hline $1 \mathrm{H}-2,62$ & 2.12 & 80.42 \\
\hline $1 \mathrm{H}-2,64$ & 2.14 & 85.12 \\
\hline $1 \mathrm{H}-2,66$ & 2.16 & 92.13 \\
\hline $1 \mathrm{H}-2,68$ & 2.18 & 97.44 \\
\hline $1 \mathrm{H}-2,70$ & 2.20 & 110.44 \\
\hline $1 \mathrm{H}-2,72$ & 2.22 & 130.15 \\
\hline $1 \mathrm{H}-2,74$ & 2.24 & 140.46 \\
\hline $1 \mathrm{H}-2,76$ & 2.26 & 127.16 \\
\hline $1 \mathrm{H}-2,78$ & 2.28 & 113.17 \\
\hline $1 \mathrm{H}-2,80$ & 2.30 & 107.48 \\
\hline $1 \mathrm{H}-2,82$ & 2.32 & 108.88 \\
\hline $1 \mathrm{H}-2,84$ & 2.34 & 112.49 \\
\hline $1 \mathrm{H}-2,86$ & 2.36 & 112.20 \\
\hline $1 \mathrm{H}-2,88$ & 2.38 & 112.50 \\
\hline $1 \mathrm{H}-2,90$ & 2.40 & 112.21 \\
\hline $1 \mathrm{H}-2,92$ & 2.42 & 107.52 \\
\hline $1 \mathrm{H}-2,94$ & 2.44 & 103.93 \\
\hline $1 \mathrm{H}-2,96$ & 2.46 & 100.54 \\
\hline $1 \mathrm{H}-2,98$ & 2.48 & 94.24 \\
\hline $1 \mathrm{H}-2,100$ & 2.50 & 92.25 \\
\hline $1 \mathrm{H}-2,102$ & 2.52 & 96.26 \\
\hline $1 \mathrm{H}-2,104$ & 2.54 & 98.57 \\
\hline $1 \mathrm{H}-2,106$ & 2.56 & 99.97 \\
\hline $1 \mathrm{H}-2,108$ & 2.58 & 100.98 \\
\hline $1 \mathrm{H}-2,110$ & 2.60 & 108.59 \\
\hline $1 \mathrm{H}-2,112$ & 2.62 & 124.30 \\
\hline $1 \mathrm{H}-2,114$ & 2.64 & 135.60 \\
\hline $1 \mathrm{H}-2,116$ & 2.66 & 139.61 \\
\hline $1 \mathrm{H}-2,118$ & 2.68 & 138.62 \\
\hline $1 \mathrm{H}-2,120$ & 2.70 & 138.32 \\
\hline $1 \mathrm{H}-2,122$ & 2.72 & 140.04 \\
\hline
\end{tabular}

\begin{tabular}{|c|c|c|}
\hline $\begin{array}{l}\text { Core, section, } \\
\text { interval }(\mathrm{cm})\end{array}$ & $\begin{array}{l}\text { Depth } \\
\text { (mbsf) }\end{array}$ & $\begin{array}{c}\text { Magnetic } \\
\text { susceptibility } \\
\left(10^{-5} \mathrm{SI}\right)\end{array}$ \\
\hline $1 \mathrm{H}-2,124$ & 2.74 & 135.64 \\
\hline $1 \mathrm{H}-2,126$ & 2.76 & 129.05 \\
\hline $1 \mathrm{H}-2,128$ & 2.78 & 125.66 \\
\hline $1 \mathrm{H}-2,130$ & 2.80 & 128.06 \\
\hline $1 \mathrm{H}-2,132$ & 2.82 & 145.07 \\
\hline $1 \mathrm{H}-2,134$ & 2.84 & 151.68 \\
\hline $1 \mathrm{H}-2,136$ & 2.86 & 152.69 \\
\hline $1 \mathrm{H}-2,138$ & 2.88 & 160.09 \\
\hline $1 \mathrm{H}-2,140$ & 2.90 & 150.10 \\
\hline $1 \mathrm{H}-2,142$ & 2.92 & 153.11 \\
\hline $1 \mathrm{H}-2,144$ & 2.94 & 143.42 \\
\hline $1 \mathrm{H}-2,146$ & 2.96 & 128.43 \\
\hline $1 \mathrm{H}-2,148$ & 2.98 & 114.43 \\
\hline $1 \mathrm{H}-3,4$ & 3.04 & 101.99 \\
\hline $1 \mathrm{H}-3,8$ & 3.08 & 94.69 \\
\hline $1 \mathrm{H}-3,12$ & 3.12 & 106.99 \\
\hline $1 \mathrm{H}-3,16$ & 3.16 & 107.68 \\
\hline $1 \mathrm{H}-3,20$ & 3.20 & 103.98 \\
\hline $1 \mathrm{H}-3,24$ & 3.24 & 91.97 \\
\hline $1 \mathrm{H}-3,28$ & 3.28 & 80.27 \\
\hline $1 \mathrm{H}-3,32$ & 3.32 & 81.27 \\
\hline $1 \mathrm{H}-3,36$ & 3.36 & 79.96 \\
\hline $1 \mathrm{H}-3,40$ & 3.40 & 84.26 \\
\hline $1 \mathrm{H}-3,44$ & 3.44 & 89.96 \\
\hline $1 \mathrm{H}-3,48$ & 3.48 & 113.25 \\
\hline $1 \mathrm{H}-3,52$ & 3.52 & 73.94 \\
\hline $1 \mathrm{H}-3,56$ & 3.56 & 59.63 \\
\hline $1 \mathrm{H}-3,60$ & 3.60 & 54.92 \\
\hline $1 \mathrm{H}-3,64$ & 3.64 & 66.60 \\
\hline $1 \mathrm{H}-3,68$ & 3.68 & 62.89 \\
\hline $1 \mathrm{H}-3,72$ & 3.72 & 60.88 \\
\hline $1 \mathrm{H}-3,76$ & 3.76 & 62.16 \\
\hline $1 \mathrm{H}-3,80$ & 3.80 & 64.85 \\
\hline $1 \mathrm{H}-3,84$ & 3.84 & 65.54 \\
\hline $1 \mathrm{H}-3,88$ & 3.88 & 72.82 \\
\hline $1 \mathrm{H}-3,92$ & 3.92 & 69.81 \\
\hline $1 \mathrm{H}-3,96$ & 3.96 & 70.80 \\
\hline $1 \mathrm{H}-3,100$ & 4.00 & 85.78 \\
\hline $1 \mathrm{H}-3,104$ & 4.04 & 80.47 \\
\hline $1 \mathrm{H}-3,108$ & 4.08 & 74.75 \\
\hline $1 \mathrm{H}-3,112$ & 4.12 & 73.73 \\
\hline $1 \mathrm{H}-3,116$ & 4.16 & 77.02 \\
\hline $1 \mathrm{H}-3,120$ & 4.20 & 80.01 \\
\hline $1 \mathrm{H}-3,124$ & 4.24 & 81.99 \\
\hline $1 \mathrm{H}-3,128$ & 4.28 & 79.97 \\
\hline $1 \mathrm{H}-3,132$ & 4.32 & 72.65 \\
\hline $1 \mathrm{H}-3,136$ & 4.36 & 74.34 \\
\hline $1 \mathrm{H}-3,140$ & 4.40 & 76.33 \\
\hline $1 \mathrm{H}-3,144$ & 4.44 & 71.61 \\
\hline $1 \mathrm{H}-3,148$ & 4.48 & 52.29 \\
\hline $1 \mathrm{H}-4,4$ & 4.54 & 60.70 \\
\hline $1 \mathrm{H}-4,8$ & 4.58 & 60.31 \\
\hline $1 \mathrm{H}-4,12$ & 4.62 & 73.71 \\
\hline $1 \mathrm{H}-4,16$ & 4.66 & 96.71 \\
\hline $1 \mathrm{H}-4,20$ & 4.70 & 147.01 \\
\hline $1 \mathrm{H}-4,24$ & 4.74 & 117.71 \\
\hline $1 \mathrm{H}-4,28$ & 4.78 & 73.71 \\
\hline $1 \mathrm{H}-4,32$ & 4.82 & 72.71 \\
\hline $1 \mathrm{H}-4,36$ & 4.86 & 66.02 \\
\hline $1 \mathrm{H}-4,40$ & 4.90 & 63.72 \\
\hline $1 \mathrm{H}-4,44$ & 4.94 & 63.72 \\
\hline $1 \mathrm{H}-4,48$ & 4.98 & 61.02 \\
\hline $1 \mathrm{H}-4,52$ & 5.02 & 76.33 \\
\hline $1 \mathrm{H}-4,56$ & 5.06 & 81.03 \\
\hline
\end{tabular}

Note: Only a portion of this table appears here. The complete table is available in ASCII. 
Table T4. Edited GRA bulk density measurements and calculated dry bulk densities, Site 1165.

\begin{tabular}{|c|c|c|c|c|c|c|c|}
\hline $\begin{array}{l}\text { Core, section, } \\
\text { interval }(\mathrm{cm})\end{array}$ & $\begin{array}{l}\text { Depth } \\
\text { (mbsf) }\end{array}$ & $\begin{array}{l}\text { Bulk } \\
\text { density } \\
\left(\mathrm{g} / \mathrm{cm}^{3}\right)\end{array}$ & $\begin{array}{c}\text { Dry } \\
\text { density } \\
\left(\mathrm{g} / \mathrm{cm}^{3}\right)\end{array}$ & $\begin{array}{l}\text { Core, section, } \\
\text { interval }(\mathrm{cm})\end{array}$ & $\begin{array}{l}\text { Depth } \\
\text { (mbsf) }\end{array}$ & $\begin{array}{c}\text { Bulk } \\
\text { density } \\
\left(\mathrm{g} / \mathrm{cm}^{3}\right)\end{array}$ & $\begin{array}{c}\text { Dry } \\
\text { density } \\
\left(\mathrm{g} / \mathrm{cm}^{3}\right)\end{array}$ \\
\hline 188-1165B- & & & & $1 \mathrm{H}-2,30$ & 1.80 & 1.493 & 0.781 \\
\hline $1 \mathrm{H}-1,12$ & 0.12 & 1.427 & 0.679 & $1 \mathrm{H}-2,32$ & 1.82 & 1.496 & 0.786 \\
\hline $1 \mathrm{H}-1,14$ & 0.14 & 1.424 & 0.675 & $1 \mathrm{H}-2,34$ & 1.84 & 1.526 & 0.832 \\
\hline $1 \mathrm{H}-1,16$ & 0.16 & 1.429 & 0.682 & $1 \mathrm{H}-2,36$ & 1.86 & 1.575 & 0.908 \\
\hline $1 \mathrm{H}-1,18$ & 0.18 & 1.457 & 0.726 & $1 \mathrm{H}-2,38$ & 1.88 & 1.554 & 0.875 \\
\hline $1 \mathrm{H}-1,20$ & 0.20 & 1.418 & 0.665 & $1 \mathrm{H}-2,40$ & 1.90 & 1.531 & 0.840 \\
\hline $1 \mathrm{H}-1,22$ & 0.22 & 1.432 & 0.687 & $1 \mathrm{H}-2,42$ & 1.92 & 1.521 & 0.824 \\
\hline $1 \mathrm{H}-1,24$ & 0.24 & 1.405 & 0.645 & $1 \mathrm{H}-2,44$ & 1.94 & 1.477 & 0.757 \\
\hline $1 \mathrm{H}-1,26$ & 0.26 & 1.420 & 0.669 & $1 \mathrm{H}-2,46$ & 1.96 & 1.495 & 0.784 \\
\hline $1 \mathrm{H}-1,28$ & 0.28 & 1.600 & 0.946 & $1 \mathrm{H}-2,48$ & 1.98 & 1.523 & 0.827 \\
\hline $1 \mathrm{H}-1,32$ & 0.32 & 1.466 & 0.740 & $1 \mathrm{H}-2,50$ & 2.00 & 1.521 & 0.824 \\
\hline $1 \mathrm{H}-1,34$ & 0.34 & 1.434 & 0.690 & $1 \mathrm{H}-2,52$ & 2.02 & 1.462 & 0.733 \\
\hline $1 \mathrm{H}-1,36$ & 0.36 & 1.469 & 0.744 & $1 \mathrm{H}-2,54$ & 2.04 & 1.503 & 0.797 \\
\hline $1 \mathrm{H}-1,38$ & 0.38 & 1.416 & 0.662 & $1 \mathrm{H}-2,56$ & 2.06 & 1.523 & 0.827 \\
\hline $1 \mathrm{H}-1,42$ & 0.42 & 1.410 & 0.653 & $1 \mathrm{H}-2,58$ & 2.08 & 1.492 & 0.780 \\
\hline $1 \mathrm{H}-1,44$ & 0.44 & 1.416 & 0.662 & $1 \mathrm{H}-2,60$ & 2.10 & 1.531 & 0.840 \\
\hline $1 \mathrm{H}-1,46$ & 0.46 & 1.423 & 0.673 & $1 \mathrm{H}-2,62$ & 2.12 & 1.521 & 0.824 \\
\hline $1 \mathrm{H}-1,52$ & 0.52 & 1.403 & 0.642 & $1 \mathrm{H}-2,64$ & 2.14 & 1.512 & 0.811 \\
\hline $1 \mathrm{H}-1,54$ & 0.54 & 1.403 & 0.642 & $1 \mathrm{H}-2,66$ & 2.16 & 1.612 & 0.965 \\
\hline $1 \mathrm{H}-1,60$ & 0.60 & 1.408 & 0.650 & $1 \mathrm{H}-2,68$ & 2.18 & 1.564 & 0.891 \\
\hline $1 \mathrm{H}-1,70$ & 0.70 & 1.433 & 0.689 & $1 \mathrm{H}-2,70$ & 2.20 & 1.639 & 1.007 \\
\hline $1 \mathrm{H}-1,72$ & 0.72 & 1.430 & 0.684 & $1 \mathrm{H}-2,72$ & 2.22 & 1.653 & 1.028 \\
\hline $1 \mathrm{H}-1,74$ & 0.74 & 1.425 & 0.676 & $1 \mathrm{H}-2,74$ & 2.24 & 1.647 & 1.019 \\
\hline $1 \mathrm{H}-1,76$ & 0.76 & 1.457 & 0.726 & $1 \mathrm{H}-2,76$ & 2.26 & 1.617 & 0.973 \\
\hline $1 \mathrm{H}-1,78$ & 0.78 & 1.417 & 0.664 & $1 \mathrm{H}-2,78$ & 2.28 & 1.607 & 0.957 \\
\hline $1 \mathrm{H}-1,80$ & 0.80 & 1.429 & 0.682 & $1 \mathrm{H}-2,80$ & 2.30 & 1.626 & 0.986 \\
\hline $1 \mathrm{H}-1,82$ & 0.82 & 1.439 & 0.698 & $1 \mathrm{H}-2,82$ & 2.32 & 1.647 & 1.019 \\
\hline $1 \mathrm{H}-1,84$ & 0.84 & 1.426 & 0.678 & $1 \mathrm{H}-2,84$ & 2.34 & 1.592 & 0.934 \\
\hline $1 \mathrm{H}-1,86$ & 0.86 & 1.459 & 0.729 & $1 \mathrm{H}-2,86$ & 2.36 & 1.658 & 1.036 \\
\hline $1 \mathrm{H}-1,88$ & 0.88 & 1.429 & 0.682 & $1 \mathrm{H}-2,88$ & 2.38 & 1.597 & 0.942 \\
\hline $1 \mathrm{H}-1,90$ & 0.90 & 1.440 & 0.699 & $1 \mathrm{H}-2,90$ & 2.40 & 1.608 & 0.959 \\
\hline $1 \mathrm{H}-1,92$ & 0.92 & 1.412 & 0.656 & $1 \mathrm{H}-2,92$ & 2.42 & 1.569 & 0.898 \\
\hline $1 \mathrm{H}-1,94$ & 0.94 & 1.442 & 0.702 & $1 \mathrm{H}-2,94$ & 2.44 & 1.617 & 0.973 \\
\hline $1 \mathrm{H}-1,96$ & 0.96 & 1.431 & 0.686 & $1 \mathrm{H}-2,96$ & 2.46 & 1.611 & 0.963 \\
\hline $1 \mathrm{H}-1,98$ & 0.98 & 1.429 & 0.682 & $1 \mathrm{H}-2,98$ & 2.48 & 1.553 & 0.874 \\
\hline $1 \mathrm{H}-1,100$ & 1.00 & 1.425 & 0.676 & $1 \mathrm{H}-2,100$ & 2.50 & 1.558 & 0.882 \\
\hline $1 \mathrm{H}-1,102$ & 1.02 & 1.400 & 0.638 & $1 \mathrm{H}-2,102$ & 2.52 & 1.557 & 0.880 \\
\hline $1 \mathrm{H}-1,104$ & 1.04 & 1.402 & 0.641 & $1 \mathrm{H}-2,104$ & 2.54 & 1.566 & 0.894 \\
\hline $1 \mathrm{H}-1,108$ & 1.08 & 1.422 & 0.672 & $1 \mathrm{H}-2,106$ & 2.56 & 1.594 & 0.937 \\
\hline $1 \mathrm{H}-1,110$ & 1.10 & 1.403 & 0.642 & $1 \mathrm{H}-2,108$ & 2.58 & 1.579 & 0.914 \\
\hline $1 \mathrm{H}-1,116$ & 1.16 & 1.421 & 0.670 & $1 \mathrm{H}-2,110$ & 2.60 & 1.581 & 0.917 \\
\hline $1 \mathrm{H}-1,120$ & 1.20 & 1.441 & 0.701 & $1 \mathrm{H}-2,112$ & 2.62 & 1.613 & 0.966 \\
\hline $1 \mathrm{H}-1,122$ & 1.22 & 1.436 & 0.693 & $1 \mathrm{H}-2,114$ & 2.64 & 1.628 & 0.990 \\
\hline $1 \mathrm{H}-1,124$ & 1.24 & 1.459 & 0.729 & $1 \mathrm{H}-2,116$ & 2.66 & 1.627 & 0.988 \\
\hline $1 \mathrm{H}-1,126$ & 1.26 & 2.140 & 1.780 & $1 \mathrm{H}-2,118$ & 2.68 & 1.592 & 0.934 \\
\hline $1 \mathrm{H}-1,128$ & 1.28 & 2.200 & 1.872 & $1 \mathrm{H}-2,120$ & 2.70 & 1.633 & 0.997 \\
\hline $1 \mathrm{H}-1,130$ & 1.30 & 1.473 & 0.750 & $1 \mathrm{H}-2,122$ & 2.72 & 1.640 & 1.008 \\
\hline $1 \mathrm{H}-1,132$ & 1.32 & 1.439 & 0.698 & $1 \mathrm{H}-2,124$ & 2.74 & 1.635 & 1.000 \\
\hline $1 \mathrm{H}-1,134$ & 1.34 & 1.454 & 0.721 & $1 \mathrm{H}-2,126$ & 2.76 & 1.590 & 0.931 \\
\hline $1 \mathrm{H}-1,136$ & 1.36 & 1.427 & 0.679 & $1 \mathrm{H}-2,128$ & 2.78 & 1.606 & 0.956 \\
\hline $1 \mathrm{H}-1,138$ & 1.38 & 1.426 & 0.678 & $1 \mathrm{H}-2,130$ & 2.80 & 1.548 & 0.866 \\
\hline $1 \mathrm{H}-1,140$ & 1.40 & 1.458 & 0.727 & $1 \mathrm{H}-2,132$ & 2.82 & 1.686 & 1.079 \\
\hline $1 \mathrm{H}-1,142$ & 1.42 & 1.460 & 0.730 & $1 \mathrm{H}-2,134$ & 2.84 & 1.605 & 0.954 \\
\hline $1 \mathrm{H}-1,144$ & 1.44 & 1.450 & 0.715 & $1 \mathrm{H}-2,136$ & 2.86 & 1.608 & 0.959 \\
\hline $1 \mathrm{H}-1,146$ & 1.46 & 1.442 & 0.702 & $1 \mathrm{H}-2,138$ & 2.88 & 1.666 & 1.048 \\
\hline $1 \mathrm{H}-1,148$ & 1.48 & 1.470 & 0.746 & $1 \mathrm{H}-2,140$ & 2.90 & 1.585 & 0.923 \\
\hline $1 \mathrm{H}-2,6$ & 1.56 & 1.409 & 0.652 & $1 \mathrm{H}-2,142$ & 2.92 & 1.704 & 1.107 \\
\hline $1 \mathrm{H}-2,8$ & 1.58 & 1.405 & 0.645 & $1 \mathrm{H}-2,144$ & 2.94 & 1.555 & 0.877 \\
\hline $1 \mathrm{H}-2,10$ & 1.60 & 1.419 & 0.667 & $1 \mathrm{H}-2,146$ & 2.96 & 1.592 & 0.934 \\
\hline $1 \mathrm{H}-2,12$ & 1.62 & 1.442 & 0.702 & $1 \mathrm{H}-2,148$ & 2.98 & 1.632 & 0.996 \\
\hline $1 \mathrm{H}-2,14$ & 1.64 & 1.435 & 0.692 & $1 \mathrm{H}-3,2$ & 3.02 & 1.564 & 0.891 \\
\hline $1 \mathrm{H}-2,16$ & 1.66 & 1.440 & 0.699 & $1 \mathrm{H}-3,4$ & 3.04 & 1.542 & 0.857 \\
\hline $1 \mathrm{H}-2,18$ & 1.68 & 1.451 & 0.716 & $1 \mathrm{H}-3,6$ & 3.06 & 1.558 & 0.882 \\
\hline $1 \mathrm{H}-2,20$ & 1.70 & 1.492 & 0.780 & $1 \mathrm{H}-3,8$ & 3.08 & 1.522 & 0.826 \\
\hline $1 \mathrm{H}-2,22$ & 1.72 & 1.482 & 0.764 & $1 \mathrm{H}-3,10$ & 3.10 & 1.564 & 0.891 \\
\hline $1 \mathrm{H}-2,24$ & 1.74 & 1.486 & 0.770 & $1 \mathrm{H}-3,12$ & 3.12 & 1.585 & 0.923 \\
\hline $1 \mathrm{H}-2,26$ & 1.76 & 1.503 & 0.797 & $1 \mathrm{H}-3,14$ & 3.14 & 1.553 & 0.874 \\
\hline $1 \mathrm{H}-2,28$ & 1.78 & 1.511 & 0.809 & $1 \mathrm{H}-3,16$ & 3.16 & 1.576 & 0.909 \\
\hline
\end{tabular}

\begin{tabular}{|c|c|c|c|}
\hline $\begin{array}{l}\text { Core, section, } \\
\text { interval }(\mathrm{cm})\end{array}$ & $\begin{array}{l}\text { Depth } \\
\text { (mbsf) }\end{array}$ & $\begin{array}{c}\text { Bulk } \\
\text { density } \\
\left(\mathrm{g} / \mathrm{cm}^{3}\right)\end{array}$ & $\begin{array}{c}\text { Dry } \\
\text { density } \\
\left(\mathrm{g} / \mathrm{cm}^{3}\right)\end{array}$ \\
\hline $1 \mathrm{H}-3,18$ & 3.18 & 1.553 & 0.874 \\
\hline $1 \mathrm{H}-3,20$ & 3.20 & 1.540 & 0.854 \\
\hline $1 \mathrm{H}-3,22$ & 3.22 & 1.517 & 0.818 \\
\hline $1 \mathrm{H}-3,24$ & 3.24 & 1.508 & 0.804 \\
\hline $1 \mathrm{H}-3,26$ & 3.26 & 1.481 & 0.763 \\
\hline $1 \mathrm{H}-3,28$ & 3.28 & 1.532 & 0.841 \\
\hline $1 \mathrm{H}-3,30$ & 3.30 & 1.518 & 0.820 \\
\hline $1 \mathrm{H}-3,32$ & 3.32 & 1.562 & 0.888 \\
\hline $1 \mathrm{H}-3,34$ & 3.34 & 1.516 & 0.817 \\
\hline $1 \mathrm{H}-3,36$ & 3.36 & 1.562 & 0.888 \\
\hline $1 \mathrm{H}-3,38$ & 3.38 & 1.548 & 0.866 \\
\hline $1 \mathrm{H}-3,40$ & 3.40 & 1.553 & 0.874 \\
\hline $1 \mathrm{H}-3,42$ & 3.42 & 1.603 & 0.951 \\
\hline $1 \mathrm{H}-3,44$ & 3.44 & 1.563 & 0.889 \\
\hline $1 \mathrm{H}-3,46$ & 3.46 & 1.568 & 0.897 \\
\hline $1 \mathrm{H}-3,48$ & 3.48 & 1.538 & 0.851 \\
\hline $1 \mathrm{H}-3,50$ & 3.50 & 1.586 & 0.925 \\
\hline $1 \mathrm{H}-3,52$ & 3.52 & 1.521 & 0.824 \\
\hline $1 \mathrm{H}-3,54$ & 3.54 & 1.488 & 0.773 \\
\hline $1 \mathrm{H}-3,56$ & 3.56 & 1.489 & 0.775 \\
\hline $1 \mathrm{H}-3,58$ & 3.58 & 1.475 & 0.753 \\
\hline $1 \mathrm{H}-3,60$ & 3.60 & 1.527 & 0.834 \\
\hline $1 \mathrm{H}-3,62$ & 3.62 & 1.525 & 0.831 \\
\hline $1 \mathrm{H}-3,64$ & 3.64 & 1.514 & 0.814 \\
\hline $1 \mathrm{H}-3,66$ & 3.66 & 1.525 & 0.831 \\
\hline $1 \mathrm{H}-3,68$ & 3.68 & 1.531 & 0.840 \\
\hline $1 \mathrm{H}-3,70$ & 3.70 & 1.541 & 0.855 \\
\hline $1 \mathrm{H}-3,72$ & 3.72 & 1.510 & 0.807 \\
\hline $1 \mathrm{H}-3,74$ & 3.74 & 1.530 & 0.838 \\
\hline $1 \mathrm{H}-3,76$ & 3.76 & 1.546 & 0.863 \\
\hline $1 \mathrm{H}-3,78$ & 3.78 & 1.546 & 0.863 \\
\hline $1 \mathrm{H}-3,80$ & 3.80 & 1.518 & 0.820 \\
\hline $1 \mathrm{H}-3,82$ & 3.82 & 1.547 & 0.865 \\
\hline $1 \mathrm{H}-3,84$ & 3.84 & 1.519 & 0.821 \\
\hline $1 \mathrm{H}-3,86$ & 3.86 & 1.503 & 0.797 \\
\hline $1 \mathrm{H}-3,88$ & 3.88 & 1.503 & 0.797 \\
\hline $1 \mathrm{H}-3,90$ & 3.90 & 1.542 & 0.857 \\
\hline $1 \mathrm{H}-3,92$ & 3.92 & 1.562 & 0.888 \\
\hline $1 \mathrm{H}-3,94$ & 3.94 & 1.591 & 0.932 \\
\hline $1 \mathrm{H}-3,96$ & 3.96 & 1.558 & 0.882 \\
\hline $1 \mathrm{H}-3,98$ & 3.98 & 1.558 & 0.882 \\
\hline $1 \mathrm{H}-3,100$ & 4.00 & 1.657 & 1.034 \\
\hline $1 \mathrm{H}-3,102$ & 4.02 & 1.605 & 0.954 \\
\hline $1 \mathrm{H}-3,104$ & 4.04 & 1.560 & 0.885 \\
\hline $1 \mathrm{H}-3,106$ & 4.06 & 1.548 & 0.866 \\
\hline $1 \mathrm{H}-3,108$ & 4.08 & 1.576 & 0.909 \\
\hline $1 \mathrm{H}-3,110$ & 4.10 & 1.559 & 0.883 \\
\hline $1 \mathrm{H}-3,112$ & 4.12 & 1.566 & 0.894 \\
\hline $1 \mathrm{H}-3,114$ & 4.14 & 1.587 & 0.926 \\
\hline $1 \mathrm{H}-3,116$ & 4.16 & 1.556 & 0.878 \\
\hline $1 \mathrm{H}-3,118$ & 4.18 & 1.576 & 0.909 \\
\hline $1 \mathrm{H}-3,120$ & 4.20 & 1.551 & 0.871 \\
\hline $1 \mathrm{H}-3,122$ & 4.22 & 1.592 & 0.934 \\
\hline $1 \mathrm{H}-3,124$ & 4.24 & 1.558 & 0.882 \\
\hline $1 \mathrm{H}-3,126$ & 4.26 & 1.577 & 0.911 \\
\hline $1 \mathrm{H}-3,128$ & 4.28 & 1.564 & 0.891 \\
\hline $1 \mathrm{H}-3,130$ & 4.30 & 1.551 & 0.871 \\
\hline $1 \mathrm{H}-3,132$ & 4.32 & 1.550 & 0.869 \\
\hline $1 \mathrm{H}-3,134$ & 4.34 & 1.556 & 0.878 \\
\hline $1 \mathrm{H}-3,136$ & 4.36 & 1.549 & 0.868 \\
\hline $1 \mathrm{H}-3,138$ & 4.38 & 1.571 & 0.902 \\
\hline $1 \mathrm{H}-3,140$ & 4.40 & 1.540 & 0.854 \\
\hline $1 \mathrm{H}-3,142$ & 4.42 & 1.573 & 0.905 \\
\hline $1 \mathrm{H}-3,144$ & 4.44 & 1.554 & 0.875 \\
\hline
\end{tabular}

Note: Only a portion of this table appears here. The complete table is available in ASCII. 
J. GRÜTZNER

Data Report: Core Data, Grain-Size Analysis, AND Biogenic Silica

Table T5. Statistics of measurements.

\begin{tabular}{lcccrrrr}
\hline \multicolumn{1}{c}{ Statistic } & $\begin{array}{c}>63 \mu \mathrm{m} \\
(\%)\end{array}$ & $\begin{array}{c}63-125 \mu \mathrm{m} \\
(\%)\end{array}$ & $\begin{array}{c}125-250 \mu \mathrm{m} \\
(\%)\end{array}$ & $\begin{array}{c}>250 \mu \mathrm{m} \\
(\%)\end{array}$ & $\begin{array}{c}\text { Biogenic } \\
\text { silica } \\
(\%)\end{array}$ & $\begin{array}{c}\text { Density } \\
\left(\mathrm{g} / \mathrm{cm}^{3}\right)\end{array}$ & $\begin{array}{c}\text { Magnetic } \\
\text { susceptibility } \\
\left(10^{-5} \mathrm{SI}\right)\end{array}$ \\
\hline Number of measurements & 274 & 274 & 274 & 274 & 76 & 6026 & 3119 \\
Minimum & 0.19 & 0.04 & 0.02 & 0.01 & 4.52 & 1.400 & 5.37 \\
Maximum & 31.90 & 6.19 & 5.79 & 28.66 & 32.66 & 2.200 & 218.83 \\
Mean & 8.06 & 1.95 & 1.78 & 4.33 & 17.07 & 1.564 & 54.99 \\
Standard deviation & 5.18 & 1.14 & 1.06 & 3.54 & 5.74 & 0.063 & 31.57 \\
\hline
\end{tabular}




\section{J. GRÜTZNER}

Data Report: Core Data, Grain-Size Analysis, AND Biogenic Silica

Table T6. Correlation coefficients between sediment parameters.

\begin{tabular}{lcccc}
\hline \multicolumn{1}{c}{ Variable } & $\begin{array}{c}>250 \mu \mathrm{m} \\
(\%)\end{array}$ & $\begin{array}{c}\text { Biogenic } \\
\text { silica } \\
(\%)\end{array}$ & $\begin{array}{c}\text { Magnetic } \\
\text { susceptibility } \\
\left(10^{-5} \mathrm{SI}\right)\end{array}$ & $\begin{array}{c}\text { Density } \\
\left(\mathrm{g} / \mathrm{cm}^{3}\right)\end{array}$ \\
\hline$>250 \mu \mathrm{m}(\%)$ & 1.00 & & & \\
Biogenic silica (\%) & 0.27 & 1.00 & & \\
Magnetic susceptibility $\left(10^{-5} \mathrm{SI}\right)$ & 0.47 & -0.20 & 1.00 & \\
Density $\left(\mathrm{g} / \mathrm{cm}^{3}\right)$ & -0.14 & -0.51 & 0.12 & 1.00 \\
\hline
\end{tabular}




\section{J. GRÜTZNER}

Data Report: Core Data, Grain-Size Analysis, ANd Biogenic Silica

Table T7. Tie points used for depth to age conversion.

\begin{tabular}{rrl}
\hline $\begin{array}{r}\text { Depth } \\
\text { (mbsf) }\end{array}$ & $\begin{array}{c}\text { Age } \\
\text { (k.y.) }\end{array}$ & Method \\
\hline 19.23 & 3,040 & Magnetic \\
20.91 & 3,110 & Magnetic \\
25.96 & 3,220 & Magnetic \\
30.76 & 3,330 & Magnetic \\
36.46 & 3,580 & Magnetic \\
42.06 & 4,180 & Magnetic \\
43.19 & 4,290 & Magnetic \\
45.75 & 4,620 & Magnetic \\
46.96 & 4,800 & Magnetic \\
48.80 & 4,890 & Magnetic \\
49.12 & 4,980 & Magnetic \\
54.56 & 5,230 & Magnetic \\
73.52 & 6,570 & Magnetic \\
89.20 & 6,940 & Magnetic \\
100.32 & 7,710 & Magnetic \\
116.02 & 9,120 & Rad. \\
172.67 & 10,360 & Rad.
\end{tabular}

